\title{
The axiom of real Blackwell determinacy
}

\author{
Daisuke Ikegami · David de Kloet • \\ Benedikt Löwe
}

Received: 19 June 2011 / Accepted: 14 May 2012 / Published online: 21 June 2012

(C) The Author(s) 2012. This article is published with open access at Springerlink.com

\begin{abstract}
The theory of infinite games with slightly imperfect information has been developed for games with finitely and countably many moves. In this paper, we shift the discussion to games with uncountably many possible moves, introducing the axiom of real Blackwell determinacy $B I-A D_{\mathbb{R}}$ (as an analogue of the axiom of real determinacy $\left.A D_{\mathbb{R}}\right)$. We prove that the consistency strength of $B I-A D_{\mathbb{R}}$ is strictly greater than that of $A D$.
\end{abstract}

Keywords Blackwell games · Sharps · Consistency strength · Real determinacy

Mathematics Subject Classification $\quad$ 03E60 $\cdot$ 03E15 $\cdot 03 E 35 \cdot 91 \mathrm{~A} 05 \cdot 91 \mathrm{~A} 44$

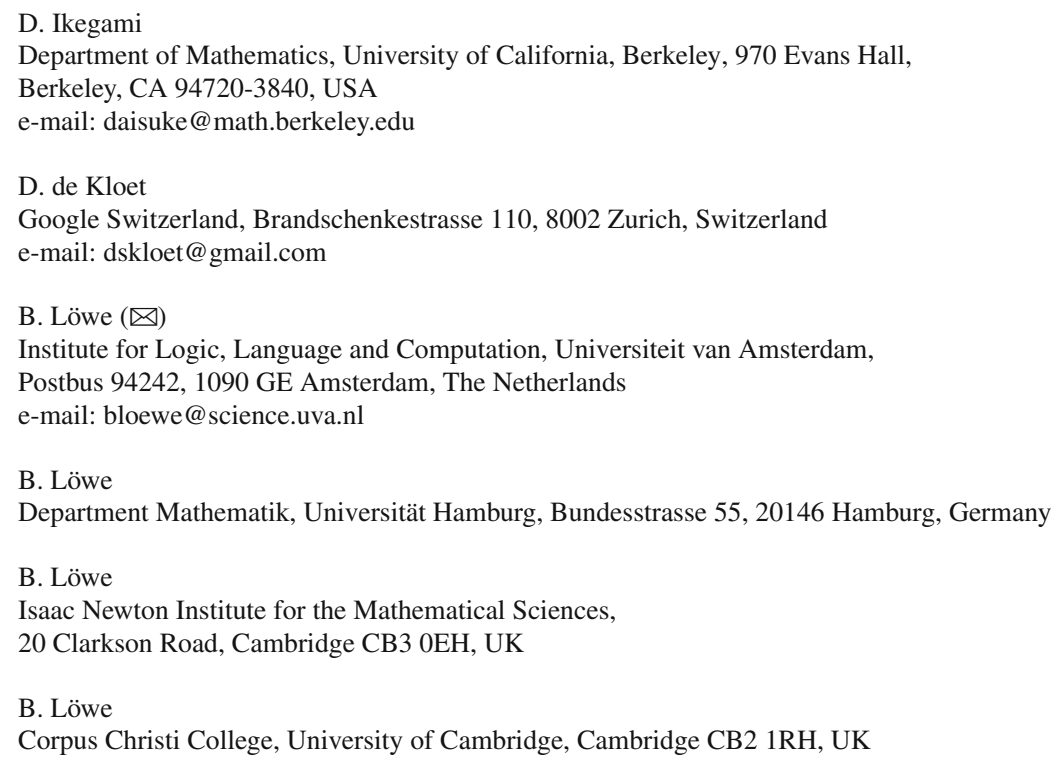




\section{Introduction and background}

Infinite perfect information games (called Gale-Stewart games after [4]) play a central rôle in the foundations of mathematics via the investigation of so-called determinacy axioms, among them the Axiom of Determinacy AD and the Axiom of Real Determinacy $A_{\mathbb{R}}$. In spite of the fact that these two axioms contradict the axiom of choice, their foundational significance can hardly be overestimated.

Blackwell games are the analogue of Gale-Stewart games without perfect information; in these games, we are restricting the lack of perfect information to an infinite sequence of simultaneously made moves (cf. Footnote 5). They were introduced by Blackwell in 1969 [2], and dubbed "games with slightly imperfect information" by him in [3]. These games allowed new proofs of known consequences of determinacy. ${ }^{1}$ In [13], Martin proved that in most cases, Blackwell determinacy axioms follow from the corresponding determinacy axioms. Martin conjectured that they are equivalent, and many instances of equivalence have been shown. ${ }^{2}$ However, the general question, and in particular the most intriguing instance, viz. whether $A D$ and the axiom of Blackwell determinacy BI-AD are equivalent, remain open.

In this paper, we turn to the other mentioned determinacy axioms, the stronger $A D_{\mathbb{R}}$ and its Blackwell analogues. We shall introduce the Axiom of Real Blackwell Determinacy $\mathrm{BI}-\mathrm{AD}_{\mathbb{R}}$ and investigate its relationship to $A \mathrm{D}_{\mathbb{R}}$. The axiom of real determinacy has been studied by Solovay in his masterful analysis [18]. Its Blackwell analogues were introduced in [9] in two variants, the countable support variant and the Euclidean variant. While we give the definition of both variants below, we shall only be concerned with the countable support variant here, and denote it by BI-AD $D_{\mathbb{R}}$. We follow Solovay's lead and provide the results analogous to [18] for $B I-A D_{\mathbb{R}}$. Our main result is:

Main Theorem 1 Assume $\mathrm{BI}-\mathrm{AD}_{\mathbb{R}}$. Then there is a fine normal measure on $\wp_{\omega_{1}}(\mathbb{R})$, and hence $\aleph_{1}$ is $<\Theta$-supercompact and $\mathbb{R}^{\#}$ exists, where $\Theta$ is the supremum of the ordinals which are surjective images of the reals. In particular, the consistency strength of $\mathrm{BI}-\mathrm{AD}_{\mathbb{R}}$ is strictly greater than that of $\mathrm{AD}$.

The paper is organized as follows: We first give all necessary definitions to make the proof of Main Theorem 1 self-contained (Sect. 2). In Sect. 3, we reduce the Main Theorem to the existence of a fine normal measure, and then, in Sects. 4 and 5, we give two different proofs for the Main Theorem by proving the existence of a fine normal measure. In the concluding Sect.6, we discuss some further results without detailed proofs and open questions. ${ }^{3}$

\footnotetext{
${ }^{1}$ E.g., Vervoort's proof of Lebesgue measurability from Blackwell determinacy [19, Theorem 4.3] inspired Martin's derived proof in [14].

2 E.g., [15] and Martin's proof of $\Pi_{1}^{1}$ determinacy presented in [11, Corollary 3.9].

3 We should like to point the reader interested in more background to the survey paper [12] containing a more detailed discussion of the various versions of Blackwell determinacy axioms and a proof of the fact that $\mathrm{BI}-\mathrm{AD}_{\mathbb{R}}$ does not follow from BI-AD (Corollary 4).
} 


\section{Definitions}

\subsection{Blackwell determinacy}

We are using standard notation from set theory and assume familiarity with descriptive set theory throughout the paper. As usual in set theory, we shall be working on Baire space ${ }^{\omega} \omega$ instead of the ordinary real numbers. Throughout we shall work in the theory $Z F+A C_{\omega}(\mathbb{R})$. This small fragment of the axiom of choice is necessary for the definition of axioms of Blackwell determinacy. Using $A C_{\omega}(\mathbb{R})$, we can develop the basics of measure theory. If we need more than $Z F+A C_{\omega}(\mathbb{R})$ for some definitions and statements, we explicitly mention the additional axioms.

Let $X$ be a set with more than one element and assume $\operatorname{AC}_{\omega}\left({ }^{\omega} X\right)$. The case most interesting for us is $X=\mathbb{R}$. Since there is a bijection between ${ }^{\omega} \mathbb{R}$ and $\mathbb{R}$, the axioms $\mathrm{AC}_{\omega}\left({ }^{\omega} \mathbb{R}\right)$ and $\mathrm{AC}_{\omega}(\mathbb{R})$ are equivalent. $\operatorname{By} \operatorname{Prob}(X)$, we denote the set of all Borel probability measures on $X$ with countable support, i.e., the set of all Borel probability measures $p$ such that there is a countable set $C \subseteq X$ with $p(C)=1$. $^{4}$ If there is an $x \in X$ such that $p(\{x\})=1$, we call $p$ a Dirac measure. From now on, we regard $X$ as a discrete topological space and topologize ${ }^{\omega} X$ as the product space. For any finite sequence $s$ of elements in $X$, let $[s]$ be the basic open set generated by $s$, i.e., $[s]=\left\{x \in{ }^{\omega} X ; s \subseteq x\right\}$.

Let $X^{\text {Even }}\left(X^{\text {Odd }}\right)$ be the set of finite sequences in $X$ with even (odd) length. We call a function $\sigma: X^{\text {Even }} \rightarrow \operatorname{Prob}(X)$ a mixed strategy for player $I$ and a function $\tau: X^{\text {Odd }} \rightarrow \operatorname{Prob}(X)$ a mixed strategy for player II. A mixed strategy $\sigma$ is called pure if for all finite sequences $s \in \operatorname{dom}(\sigma)$, we have that $\sigma(s)$ is a Dirac measure. Pure strategies correspond to functions $\sigma: X^{\operatorname{dom}(\sigma)} \rightarrow X$, the standard type of strategies in Gale-Stewart games, and we can define the usual notions of winning strategies. Given mixed strategies $\sigma$ and $\tau$ for players I and II respectively, let $\nu(\sigma, \tau):{ }^{<\omega} X \rightarrow \operatorname{Prob}(X)$ as follows: For each finite sequence $s$ of elements in $X$,

$$
v(\sigma, \tau)(s)= \begin{cases}\sigma(s) & \text { if } \operatorname{lh}(s) \text { is even } \\ \tau(s) & \text { if } \operatorname{lh}(s) \text { is odd }\end{cases}
$$

where $\operatorname{lh}(s)$ is the length of $s$. Since some of the calculations in this paper require a lot of parentheses, let us reduce their number by convention. If $\left(x_{0}, \ldots, x_{n}\right)$ is a finite sequence, we write $\left[x_{0}, \ldots, x_{n}\right]$ for the basic open set $\left[\left(x_{0}, \ldots, x_{n}\right)\right]$. Similarly, if $x \in X$ and $\mu \in \operatorname{Prob}(X)$, we write $\mu(x)$ for $\mu(\{x\})$. Now, for each finite sequence $s$ of elements in $X$, define

$$
\mu_{\sigma, \tau}([s])=\prod_{i=0}^{\operatorname{lh}(s)-1} v(\sigma, \tau)(s\lceil i)(s(i))
$$

\footnotetext{
${ }^{4}$ We are going to amalgamate a sequence of such measures to produce a product measure on ${ }^{\omega} X$ as we construct the Lebesgue measure on ${ }^{\omega} \omega$. For this purpose, the condition of having countable support is essential.
} 
By using $\mathrm{AC}_{\omega}\left(\mathbb{R} \times{ }^{\omega} X\right)$ (which follows from $\mathrm{AC}_{\omega}\left({ }^{\omega} X\right)$ ), we can uniquely extend $\mu_{\sigma, \tau}$ to a Borel probability measure on ${ }^{\omega} X$, i.e., the probability measure whose domain is the set of all Borel sets in ${ }^{\omega} X$. Let us also use $\mu_{\sigma, \tau}$ for denoting this Borel probability measure.

Let $A$ be a subset of ${ }^{\omega} X$. A mixed strategy $\sigma$ for player I is optimal in $A$ if for any mixed strategy $\tau$ for player II, $A$ is $\mu_{\sigma, \tau}$-measurable and $\mu_{\sigma, \tau}(A)=1$. Similarly, a mixed strategy $\tau$ for player II is optimal in $A$ if for any mixed strategy $\sigma$ for player I, $A$ is $\mu_{\sigma, \tau}$-measurable and $\mu_{\sigma, \tau}(A)=0$. We say that $A$ is Blackwell determined if either player I or II has an optimal strategy in $A$. Finally, BI-AD $X$ is the statement "for any subset $A$ of ${ }^{\omega} X, A$ is Blackwell determined." 5

Remark 2 For any $X, A_{X}$ implies $B D_{-} D_{X}$. In particular, $A D_{\mathbb{R}}$ implies $B \mathrm{BI}_{\mathbb{R}}$. If there is an injective map from $X$ to $Y$, then $\mathrm{Bl}_{-}-\mathrm{AD}_{Y}$ implies $\mathrm{Bl}-\mathrm{AD}_{X}$. In particular, $\mathrm{BI}-\mathrm{AD}_{\mathbb{R}}$ implies $\mathrm{BI}-\mathrm{AD}:=\mathrm{BI}-\mathrm{AD}_{\omega}$.

\subsection{Blackwell determinacy and choice}

The third author proved in 2005 that $B I-A D_{\mathbb{R}}$ proves fragments of the axiom of choice that allow us to separate it (in terms of implication, not yet in consistency strength) from $\mathrm{BI}-\mathrm{AD}$.

Theorem 3 If $X:=Y \cup Z$ is linearly ordered and $\mathrm{AC}_{\omega}\left({ }^{\omega} X\right)$ and $\mathrm{BI}-\mathrm{AD}_{X}$ hold, then $\mathrm{AC}_{Y}(Z)$ holds.

Proof [12, Theorem 9.3].

Corollary 4 Therefore, $\mathrm{BI}-\mathrm{AD}_{\mathbb{R}}$ implies $\mathrm{AC}_{\mathbb{R}}(\mathbb{R})$, and if $\mathrm{BI}-\mathrm{AD}$ is consistent, it cannot prove $\mathrm{BI}-\mathrm{AD}_{\mathbb{R}}$.

Proof The first claim is an immediate consequence of Theorem 3. It is well-known that if $\mathbb{R}$ is not wellordered in $\mathbf{L}(\mathbb{R})$, then $\mathrm{AC}_{\mathbb{R}}(\mathbb{R})$ is false in $\mathbf{L}(\mathbb{R})$. If $\mathbf{V} \models \mathrm{BI}$-AD, then $\mathbf{L}(\mathbb{R}) \models \mathrm{Bl}$-AD and $\mathbb{R}$ is not wellordered in $\mathbf{L}(\mathbb{R})$ (because every set of reals in $\mathbf{L}(\mathbb{R})$ is Lebesgue measurable by the result of Vervoort [19, Theorem 4.3]). Thus we have a model of BI-AD $\wedge \neg$ BI-AD $\mathbb{R}_{\mathbb{R}}$.

Corollary 5 (DC) If $\mathrm{BI}-\mathrm{AD}$ implies $\mathrm{AD}$, then $\mathrm{BI}-\mathrm{AD}_{\mathbb{R}}$ implies $\mathrm{AD}_{\mathbb{R}}$.

Proof Woodin proved that under the assumption of $A D$ and $D C, A D_{\mathbb{R}}$ is equivalent to $\mathrm{AC}_{\mathbb{R}}(\mathbb{R})$ [6, Theorem 32.23]. The claim then follows directly from the assumptions and Corollary 4.

\footnotetext{
5 This formulation of Blackwell determinacy axioms does not involve imperfect information games; the original formulation due to Blackwell did, but these axioms turned out to be equivalent to the version we defined here which could be described as "perfect information determinacy with mixed strategies". The imperfect information axiom would allow the players to move simultaneously, but at each move at least one of the players would have only finitely many choices. The proof of [13] adapts to show that the perfect information axiom implies the imperfect information one. Vervoort's proof in [15] shows that optimal strategies exist for the perfect information games. For more details, cf. [12, §5].
} 


\subsection{Measures and supercompactness}

As usual, $\Theta:=\sup \{\alpha$; there is a surjection from $\mathbb{R}$ onto $\alpha\}$. Let $X$ be a set and $\kappa$ be an uncountable cardinal. As usual, we denote by $\wp_{\kappa}(X)$ the set of all subsets of $X$ with cardinality less than $\kappa$, i.e., subsets $A$ such that there is an $\alpha<\kappa$ and a surjection from $\alpha$ to $A$. Let $U$ be a set of subsets of $\wp_{\kappa}(X)$. We say that $U$ is $\kappa$-complete if $U$ is closed under intersections of less than $\kappa$ elements; we say it is non-principal if for any $a \in \wp_{\kappa}(X),\{a\} \notin U$; we say it is fine if for any $x \in X,\left\{a \in \wp_{\kappa}(X) ; x \in a\right\} \in U$; we say that $U$ is normal if for any family $\left\{A_{x} \in U ; x \in X\right\}$, the diagonal intersection $\triangle_{x \in X} A_{x}$ is in $U$ (where $\triangle_{x \in X} A_{x}=\left\{a \in \wp_{\kappa}(X) ;(\forall x \in a) a \in A_{x}\right\}$ ). We say that $U$ is a fine measure if it is a fine $\kappa$-complete ultrafilter, and we say that it is a fine normal measure if it is a fine normal $\kappa$-complete ultrafilter. It is easy to check that if there is a surjection from $X$ to $Y$, and there is a fine (normal) measure on $\wp_{\kappa}(X)$, then there is one on $\wp_{\kappa}(Y)$.

In the choice-less context, we can define a cardinal $\kappa$ to be $\lambda$-supercompact if there is a fine normal measure on $\wp_{\kappa}(\lambda)$ and to be $\lambda$-strongly compact if there is a fine measure on $\wp_{\kappa}(\lambda)$. In the ZFC-context, this is equivalent to the usual definition (cf. [6, Theorem 22.7]). It is well-known that AD implies that $\aleph_{1}$ is $\aleph_{2}$-supercompact [1].

\subsection{An alternative definition of the axiom of real Blackwell determinacy}

In [9], the second author started the investigation of the axiom of real Blackwell determinacy, and gave two alternative definitions of $B \mathrm{ILD}_{\mathbb{R}}$. The second definition was the source of the definitions of long Blackwell games in [12, §9.2]. For the sake of completeness, we give this definition here.

Instead of considering $\mathbb{R}$ as discretely topologized, we use the usual topology on $\mathbb{R}$. In this setting, we do not require strategies to have countable support. A function assigning an arbitrary Borel probability measure on $\mathbb{R}$ to each finite sequence $s$ of reals is called an E-mixed strategy. If $\sigma$ and $\tau$ are E-mixed strategies for players I and II respectively, we define

$$
v(\sigma, \tau)(s)= \begin{cases}\sigma(s) & \text { if } \operatorname{lh}(s) \text { is even } \\ \tau(s) & \text { if } \operatorname{lh}(s) \text { is odd }\end{cases}
$$

as before. If $B$ is a $k+1$-dimensional Borel set and $s \in \mathbb{R}^{k}$, we let $B_{s}:=$ $\{x ; s \frown\langle x\rangle \in B\}$. For $k \in \omega$, we define a Borel probability measure on $\mathbb{R}^{k+1}$. Define

$$
\begin{aligned}
& \mu_{\sigma, \tau}^{0}(B):=v(\sigma, \tau)(\varnothing)(B), \quad \text { and } \\
& \mu_{\sigma, \tau}^{k+1}(B):=\int_{s \in \mathbb{R}^{k}} v(\sigma, \tau)(s)\left(B_{s}\right) d \mu_{\sigma, \tau}^{k} .
\end{aligned}
$$

Clearly, the sequence of measures $\left\langle\mu_{\sigma, \tau}^{k} ; k \in \omega\right\rangle$ coheres, i.e.,

$$
\mu_{\sigma, \tau}^{k+1}(B \times \mathbb{R})=\mu_{\sigma, \tau}^{k}(B),
$$


and thus generate a Borel measure $\mu_{\sigma, \tau}^{\mathrm{E}}$ on ${ }^{\omega} \mathbb{R}$ by the Kolmogorov consistency theorem.

For a subset $A \subseteq{ }^{\omega} \mathbb{R}$, we define the other notions as before: An E-mixed strategy $\sigma$ for player I is E-optimal in $A$ if for any E-mixed strategy $\tau$ for player II, $A$ is $\mu_{\sigma, \tau}^{\mathrm{E}}$-measurable and $\mu_{\sigma, \tau}^{\mathrm{E}}(A)=1$. Similarly, an E-mixed strategy $\tau$ for player II is E-optimal in $A$ if for any E-mixed strategy $\sigma$ for player I, $A$ is $\mu_{\sigma, \tau}^{\mathrm{E}}$-measurable and $\mu_{\sigma, \tau}^{\mathrm{E}}(A)=0$. We say that $A$ is Euclidean Blackwell determined if either player I or II has an E-optimal strategy in $A$. Finally, EBI-AD $\mathbb{R}_{\mathbb{R}}$ is the statement "for any subset $A$ of ${ }^{\omega} \mathbb{R}, A$ is Euclidean Blackwell determined".

It is easy to see that $E B I-A D_{\mathbb{R}}$ implies BI-AD. The proofs of Sect. 2.2 all go through under the assumption of EBI-AD $\mathbb{R}$, so it is strictly stronger than BI-AD. We do not know what the exact relationship between $E B I-A D_{\mathbb{R}}$ and $B I-A D_{\mathbb{R}}$ is.

\section{Solovay's analysis: reducing the main theorem to the existence of a fine normal measure}

In [18], Solovay provided the foundations of the theory of $A D_{\mathbb{R}}$ while at the same time gaining some understanding of the relationship between determinacy axioms and fragments of the axiom of choice. The set $\mathbb{R}^{\#}$ encodes a truth definition of $\mathbf{L}(\mathbb{R})$ in the same sense that $0^{\#}$ encodes a truth definition of $\mathbf{L}$. It yields a proper class of indiscernibles for $\mathbf{L}(\mathbb{R})$ such that they and the reals have $\mathbf{L}(\mathbb{R})$ as their Skolem hull in $\mathbf{L}(\mathbb{R})$ while it can be recovered from such a class of indiscernibles (for details, cf. [18, §4]). In particular, if $\mathbf{L}(\mathbb{R}) \models T$, then the existence of $\mathbb{R}^{\#}$ implies Cons $(T)$, and thus by Gödel's incompleteness theorem, $T \nvdash \nvdash^{\prime \prime} \mathbb{R}^{\#}$ exists".

Fix $A \subseteq \wp_{\omega_{1}}(\mathbb{R})$ and consider the following game $\mathcal{G}_{A}$ : Players alternately play reals producing an infinite sequence $\vec{x}=\left(x_{i} ; i \in \omega\right)$. Then player II wins the game $\mathcal{G}_{A}$ if $\left\{x_{n} ; n \in \omega\right\} \in A$, otherwise player I wins.

The following two theorems are the core of Solovay's analysis:

Theorem 6 (Solovay) The axiom $A D_{\mathbb{R}}$ implies that there is a fine normal measure on $\wp \omega_{1}(\mathbb{R})$.

Proof Solovay defined a family $U \subseteq \wp\left(\wp_{\omega_{1}}(\mathbb{R})\right)$ as follows:

$A \in U$ if and only if player II has a winning strategy in $\mathcal{G}_{A}$.

Assuming $\mathrm{AD}_{\mathbb{R}}$, Solovay then proved that this is a fine normal measure (cf. [18, Lemma 3.1]).

Theorem 7 (Solovay) Suppose there is a fine normal measure on $\wp_{\omega_{1}}(\mathbb{R})$. Then $\mathbb{R}^{\#}$ exists.

Proof [18, Lemma 4.1 and Theorem 4.4].

From Theorems 6 and 7, Solovay deduced that $A D_{\mathbb{R}}$ is strictly stronger (in terms of consistency strength) than $A D$ (as $\mathbf{L}(\mathbb{R}) \models A D$ ). In Sects. 4 and 5, we shall give two proofs of the existence of a fine normal measure on $\wp_{\omega_{1}}(\mathbb{R})$ under the assumption 
of $B$ I-AD $D_{\mathbb{R}}$. If $\kappa<\Theta$, then there is a surjection from $\mathbb{R}$ onto $\kappa$ witnessing this. This surjection allows us to pull back the fine normal measure on $\wp_{\omega_{1}}(\mathbb{R})$ to $\wp_{\omega_{1}}(\kappa)$, and so $\omega_{1}$ is $\kappa$-supercompact for every $\kappa<\Theta$.

Theorem 8 (Martin, Neeman, Vervoort) If $\mathbf{V}=\mathbf{L}(\mathbb{R})$ and $\mathrm{BI}-\mathrm{AD}$ holds, then $\mathrm{AD}$ holds.

Assume that $\mathrm{BI}-\mathrm{AD} \mathrm{D}_{\mathbb{R}}$ holds. By Remark 2, we also have BI-AD which pulls back to $\mathbf{L}(\mathbb{R})$, so $\mathbf{L}(\mathbb{R}) \models B I-A D$, and hence $\mathbf{L}(\mathbb{R}) \models A D$ by Theorem 8 . But by Theorem 7 , we get $\mathbb{R}^{\#}$, and thus Cons(AD).

\section{Existence of a fine normal measure: the first proof}

In this section, we shall give the first proof of the existence of a fine normal measure on $\wp_{\omega_{1}}(\mathbb{R})$. We assume $B$ I $-\mathrm{D}_{\mathbb{R}}$ and prove the (pure strategy) determinacy of games whose payoff sets are range invariant.

A subset $A$ of ${ }^{\omega} \mathbb{R}$ is range invariant if for any $\vec{x}$ and $\vec{y}$ in ${ }^{\omega} \mathbb{R}$ with $\operatorname{ran}(\vec{x})=$ $\operatorname{ran}(\vec{y}), \vec{x} \in A$ if and only if $\vec{y} \in A$. Note that the games $\mathcal{G}_{A}$ defined in Sect. 3 are range invariant.

Lemma 9 Assume $B I-A D_{\mathbb{R}}$. Then every range invariant subset of ${ }^{\omega} \mathbb{R}$ is determined.

Proof (of Lemma 9) Let $A$ be an range invariant subset of ${ }^{\omega} \mathbb{R}$. We show that if there is an optimal strategy for player I in $A$, then so is a (pure) winning strategy for player I in $A .{ }^{6}$ The case for player II is similar and we shall skip it.

Let us first introduce some notation. Given a function $f:<\omega \mathbb{R} \rightarrow \mathbb{R}, a \in \wp_{\omega_{1}}(\mathbb{R})$ is closed under $f$ if $f^{\text {" }<\omega} a \subseteq a$. For a pure strategy $\sigma: \mathbb{R}^{\text {Even }} \rightarrow \mathbb{R}$ for player I, $a \in \wp_{\omega_{1}}(\mathbb{R})$ is closed under $\sigma$ if $\sigma$ " $a^{\text {Even }} \subseteq a$. For a function $F:{ }^{<\omega} \mathbb{R} \rightarrow \wp_{\omega_{1}}(\mathbb{R})$, $a \in \wp_{\omega_{1}}(\mathbb{R})$ is closed under $F$ if for any $s \in{ }^{<\omega} a, F(s) \subseteq a$.

Claim 10 The following are equivalent:

1. There is a winning strategy for player I in A,

2. there is a function $F:{ }^{<\omega} \mathbb{R} \rightarrow \wp_{\omega_{1}}(\mathbb{R})$ such that if a is closed under $F$, then any enumeration of a belongs to $A$, and

3. there is a function $f:{ }^{<\omega} \mathbb{R} \rightarrow \mathbb{R}$ such that if a is closed under $f$, then any enumeration of a belongs to $A$.

Proof (of Claim 10) The direction from (1) to (2) is easy to see: Given such a $\sigma$, let $f:{ }^{<\omega} \mathbb{R} \rightarrow \mathbb{R}$ be any extension of $\sigma$ and for $s \in{ }^{<\omega} \mathbb{R}$, let $F(s)=\{f(s)\}$. This $F$ works for our purpose.

We show the direction from (2) to (3): Given such an $F$, it suffices to show that there is an $f$ such that if $a$ is closed under $f$, then $a$ is also closed under $F$. Fix a bijection $\pi: \mathbb{R} \rightarrow{ }^{\omega} \mathbb{R}$. Let $g:{ }^{<} \mathbb{R} \rightarrow \mathbb{R}$ be such that $\operatorname{ran}(\pi(g(s)))=F(s)$ for each $s$ (this is possible because every relation on the reals can be uniformized by a function by Corollary 4). Let $h:{ }^{<\omega} \mathbb{R} \rightarrow \mathbb{R}$ be such that $h(s)=\pi(s(0))(\operatorname{lh}(s)-1)$ when $s \neq \varnothing$, where $\operatorname{lh}(s)$ is the length of $s$, if $s=\varnothing$ let $h(s)$ be an arbitrary real.

\footnotetext{
${ }^{6}$ Recall that pure strategies are strategies for Gale-Stewart games.
} 
It is easy to see that if $a$ is closed under $g$ and $h$, then so is under $F$ : Fix an $s \in{ }^{<\omega} a$. We have to show that $F(s) \subseteq a$. Consider $g(s)$. By the closure under $g, g(s)$ is in $a$. By choice of $g$, we know that $\operatorname{ran}(\pi(g(s)))=F(s)$, so it is enough to show that $x$ is in $a$ for any $x$ in $\operatorname{ran}(\pi(g(s))$. Suppose $x$ is the $n$th bit of $\pi(g(s))$. Consider the finite sequence $t=(g(s), \ldots, g(s))$ of length $n+1$. Then $h(t)=\pi(t(0))(\operatorname{lh}(t))=$ $\pi(g(s))(n)=x$. But $g(s)$ is in $a$ and $a$ was closed under $h$, so $x$ is in $a$.

Now it is easy to construct an $f$ such that if $a$ is closed under $f$, then so is under $g$ and $h$.

We finally show the direction from (3) to (1). Given such an $f$, we can arrange a pure strategy $\sigma$ for player I such that if $x$ is a run of the game following $\sigma$, then the range of $x$ is closed under $f$ : Given a finite sequence of reals $\left(a_{0}, \ldots, a_{2 n-1}\right)$, consider the set of all finite sequences $s$ from elements of $\left\{a_{0}, \cdots a_{2 n-1}\right\}$ and all the values $f(s)$ from this set. What we should arrange is to choose $\sigma\left(a_{0}, \ldots, a_{2 n-1}\right)$ in such a way that the range of any run of the game via $\sigma$ will cover all such values $f(s)$ when $\left(a_{0}, \ldots, a_{2 n-1}\right)$ is a finite initial segment of the run for any $n$ in $\omega$ moves. But this is possible by a standard book-keeping argument. By the property of $f$, this implies that $x$ is in $A$ and hence $\sigma$ is winning for player I.

$\square$ (Claim 10)

Therefore, to obtain a winning strategy for player I in $A$, it suffices to show (2) in Claim 10, i.e., there is a function $F:{ }^{<\omega} \mathbb{R} \rightarrow \wp_{\omega_{1}}(\mathbb{R})$ such that if $a$ is closed under $F$, then any enumeration of $a$ belongs to $A$. Let $\sigma$ be an optimal strategy for player I in $A$. Let $F$ be as follows:

$$
F(s)= \begin{cases}\varnothing & \text { if } \operatorname{lh}(s) \text { is odd } \\ \{y \in \mathbb{R} ; \sigma(s)(y) \neq 0\} & \text { otherwise. }\end{cases}
$$

Then $F$ is as desired: If $a$ is closed under $F$, then enumerate $a$ to be $\left\langle a_{n} ; n \in \omega\right\rangle$ and let player I follow $\sigma$ and let player II play the Dirac measure for $a_{n}$ at her $n$th move. Then the probability of the set $\left\{x \in{ }^{\omega} \mathbb{R} ; \operatorname{ran}(x)=a\right\}$ is 1 and since $\sigma$ is optimal for player $\mathrm{I}$ in $A$, there is an $x$ such that the range of $x$ is $a$ and $x$ is in $A$. But by the range invariance of $A$, any enumeration of $a$ belongs to $A$.

$\square$ (Lemma 9)

We shall now show that the family $U$ defined in Solovay's proof of Theorem 6 is a fine normal measure. Inspection of Solovay's proof shows that the games used to show that $U$ is a fine measure are all (equivalent to the games) of the type $\mathcal{G}_{A}$ which are range invariant and thus by Lemma 9 , determined. Therefore, $U$ is a fine measure.

We shall now show that $U$ is normal. For this, let $\left\{A_{x} ; x \in \mathbb{R}\right\}$ be a family of sets in $U$. We show that $\triangle_{x \in \mathbb{R}} A_{x}$ is in $U$. Consider the following game $\mathcal{G}$ : Player I moves $x$, then player II passes, and after that, they play the game $\mathcal{G}_{A_{x}}$. This game is Blackwell determined by $\mathrm{BI}-\mathrm{AD}_{\mathbb{R}}$. Player I cannot have an optimal strategy: Since $A_{x} \in U$, player II has a winning strategy $\tau_{x}$ in $\mathcal{G}_{A_{x}}$; thus, any mixed strategy that gives positive measure to some $x$ will not play optimally against $\tau_{x}$. By Blackwell determinacy, player II has an optimal strategy, call it $\tau$. Let $F:{ }^{<\omega} \mathbb{R} \rightarrow \wp_{\omega_{1}}(\mathbb{R})$ be as follows:

$$
F(s)= \begin{cases}\varnothing & \text { if } \operatorname{lh}(s) \text { is even } \\ \{y \in \mathbb{R} ; \tau(s)(y) \neq 0\} & \text { otherwise. }\end{cases}
$$


The analogue of Claim 10 shows that in order to show that player II has a winning strategy in $\mathcal{G}_{\triangle_{x \in \mathbb{R}}} A_{x}$, we only need to show that if $a$ is closed under $F$, then $a \in \triangle_{x \in \mathbb{R}} A_{x}$.

Suppose $a=\left\{x_{i} ; i \in \omega\right\}$ is closed under $F$. Fix an arbitrary $x \in a$. We shall show that $a \in A_{x}$, by considering a run of the game $\mathcal{G}$. In this run, let player I start with $x$ and then play the Dirac measure concentrating on $x_{n}$ at the $n$th move, and let player II follow $\tau$. By construction, player I will with probability 1 only play elements of $a$, and thus, by definition of $F$ and the fact that $a$ is closed under $F$, player II will with probability 1 only play elements of $a$ either. Consequently, with probability 1 , the set produced by players I and II is just $a$. But $\tau$ was optimal for player II, so $a \in A_{x}$.

This proves that $U$ is a fine normal measure, and thus, we have finished the first proof of our Main Theorem.

\section{Existence of a fine normal measure: the second proof}

In our second proof, we shall be formulating the Blackwell analogue of Solovay's fine normal measure from Theorem 6 and prove that this is a fine normal measure.

We define a family $U \subseteq \wp\left(\wp_{\omega_{1}}(\mathbb{R})\right)$ by using the games $\mathcal{G}_{A}$ defined in the proof of Theorem 6, and defining $U$ by

$A \in U$ if and only if player II has an optimal strategy in $\mathcal{G}_{A}$.

The object $U$ is the obvious Blackwell analogue of Solovay's normal measure. In the following, we shall show that under the assumption of $B I-A D_{\mathbb{R}}$, it is a fine normal measure, thus finishing the proof.

A few properties of $U$ are obvious: For instance, we see readily that $\varnothing \notin U$ and that $\wp_{\omega_{1}}(\mathbb{R}) \in U$, as well as the fact that $U$ is closed under taking supersets. In order to see that $U$ is a fine family, fix a real $x$, and let player II play $\{x\}$ with probability 1 in her first move; this is an optimal strategy for $\mathcal{G}_{\{a ; x \in a\}}$. Note that under the assumption of $\mathrm{Bl}-\mathrm{AD}_{\mathbb{R}}$, all of the games $\mathcal{G}_{A}$ are Blackwell determined.

In order to prove the other required properties of a normal measure, we need to develop the appropriate transfer technique (as discussed and applied in [10]) for the present context. Let $\pi \subseteq \omega$ be an infinite and co-infinite set. We think of $\pi$ as the set of rounds in which player I moves. We identify $\pi$ with the increasing enumeration of its members, i.e., $\pi=\left\{\pi_{i} ; i \in \omega\right\}$. Similarly, we write $\bar{\pi}$ for the increasing enumeration of $\omega \backslash \pi$, i.e., $\omega \backslash \pi=\left\{\bar{\pi}_{i} ; i \in \omega\right\}$. For notational ease, we call $\pi$ a I-coding if no two consecutive numbers are in $\pi$ and a II-coding if no two consecutive numbers are in $\omega \backslash \pi$.

Fix $A \subseteq \wp_{\omega_{1}}(\mathbb{R})$ and define two variants of $\mathcal{G}_{A}$ with alternative orders of play as determined by $\pi$. If $\pi$ is a I-coding, the game $\mathcal{G}_{A}^{\pi, \mathrm{I}}$ is played as follows:

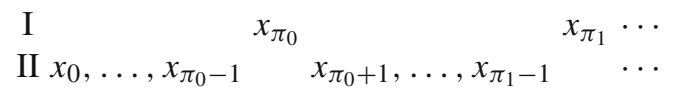

If $\pi$ is a II-coding, then we play the game $\mathcal{G}_{A}^{\pi, \text { II }}$ as follows:

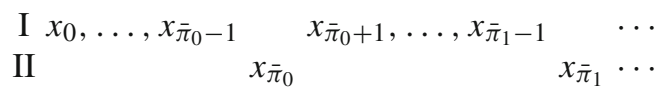


In both cases, player II wins the game if $\left\{x_{n} ; n \in \omega\right\} \in A$. Obviously, we have

$$
\mathcal{G}_{A}=\mathcal{G}_{A}^{\text {Even, II }}
$$

where Even is the set of even numbers.

Lemma 11 Let $A$ be a subset of $\wp_{\omega_{1}}(\mathbb{R})$ and $\pi$ be a I-coding. Then there is a translation $\sigma \mapsto \sigma_{\pi}$ of mixed strategies for player I such that if $\sigma$ is an optimal strategy for player I in $\mathcal{G}_{A}$, then $\sigma_{\pi}$ is an optimal strategy for player I in $\mathcal{G}_{A}^{\pi, \mathrm{I}}$.

Similarly, if $\pi$ is a II-coding, there is a translation $\tau \mapsto \tau_{\pi}$ of mixed strategies for player II such that if $\tau$ is an optimal strategy for player II in $\mathcal{G}_{A}$, then $\tau_{\pi}$ is an optimal strategy for player II in $\mathcal{G}_{A}^{\pi, \text { II }}$.

Proof We prove only the claim for the games $\mathcal{G}_{A}^{\pi, \mathrm{I}}$, the other proof being similar.

Fix a I-coding $\pi$. Let $\pi^{*}$ be the permutation of $\omega$ defined by:

$$
\begin{aligned}
\pi^{*}(2 i) & =\pi_{i} ; \\
\pi^{*}(2 i+1) & =\bar{\pi}_{i+1} .
\end{aligned}
$$

Let $h:{ }^{\omega} \mathbb{R} \rightarrow{ }^{\omega} \mathbb{R}$ be the range-preserving homeomorphism given by setting $h(\vec{x})\left(\pi^{*}(n)\right)=\vec{x}(n)$.

If $s$ is a position in $\mathcal{G}_{A}^{\pi, \mathrm{I}}$ of length $\pi_{n}$, let $g(s)$ be the corresponding position of length $2 n$ in $\mathcal{G}_{A}$, i.e., for $i<2 n$ let $g(s)(i)=s\left(\pi^{*}(i)\right)$. (In $g(s)$, player II has made only $n$ of the moves corresponding to the $\pi_{n}-n$ moves that II has made in $s$.) by

If $\sigma$ is a mixed strategy for I in $\mathcal{G}_{A}$, let $\sigma_{\pi}$ be the mixed strategy for $\mathrm{I}$ in $\mathcal{G}_{A}^{\pi, \mathrm{I}}$ given

$$
\sigma_{\pi}(s)=\sigma(g(s))
$$

We shall show that $\sigma_{\pi}$ is optimal for player I in $\mathcal{G}_{A}$ assuming $\sigma$ is optimal for player I in $\mathcal{G}_{A}^{\pi, \mathrm{I}}$. Let $\tau$ be any mixed strategy for player II in $\mathcal{G}_{A}$. We show that the set $\{\vec{x} ; \operatorname{ran}(\vec{x}) \in A\}$ has $\mu_{\sigma_{\pi}, \tau}$-measure 1 .

If $s$ is a position in $\mathcal{G}_{A}$ of length $2 n+1$, let $f(s)$ be the corresponding position of length $\bar{\pi}_{n}$ in $\mathcal{G}_{A}^{\pi, \mathrm{I}}$, let $\hat{f}(\tau)$ be the mixed strategy for II in $\mathcal{G}_{A}$ given by

$$
\hat{f}(\tau)(s)=\tau(f(s)) .
$$

By induction on length, one can easily show that, for all $s \in{ }^{<\omega} \mathbb{R}$,

$$
\mu_{\sigma, \hat{f}(\tau)}[s]=\mu_{\sigma_{\pi}, \tau}\left(h^{“}[s]\right)
$$

Since $h$ is range-preserving,

$$
\mu_{\sigma, \hat{f}(\tau)}(\{\vec{x} ; \operatorname{ran}(\vec{x}) \in A\})=\mu_{\sigma_{\pi}, \tau}(\{\vec{x} ; \operatorname{ran}(\vec{x}) \in A\})
$$


Since $\mu$ is optimal for player I in $\mathcal{G}_{A}^{\pi, \mathrm{I}}$, the left-hand side of the above formula is equal to 1 . Therefore, the set $\{\vec{x} ; \operatorname{ran}(\vec{x}) \in A\}$ has $\mu_{\sigma_{\pi}, \tau}$-measure 1 , as desired.

$\square$ (Lemma 11)

Based on Lemma 11, we can now finish the proof of the Main Theorem.

Claim 12 If $A \notin U$, then $\wp_{\omega_{1}}(\mathbb{R}) \backslash A \in U$.

Proof If player II does not have an optimal strategy in $\mathcal{G}_{A}$, then player I does. Let $\pi:=$ Odd, the set of odd numbers. Then in $\mathcal{G}_{A}^{\pi, \mathrm{I}}$, the rôles of players I and II are switched. By Lemma 11, there is an optimal strategy $\sigma_{\pi}$ for player I in the game $\mathcal{G}_{A}^{\pi, \mathrm{I}}$, but this is optimal for player II in $\mathcal{G}_{\wp_{\omega_{1}}}(\mathbb{R}) \backslash A$.

$\square($ Claim 12)

Claim 13 If $A_{1}, A_{2} \in U$, then $A_{1} \cap A_{2} \in U$.

Proof Since $A_{1}, A_{2} \in U$, there are optimal strategies $\tau_{1}$ and $\tau_{2}$ for player II in $\mathcal{G}_{A_{1}}$ and $\mathcal{G}_{A_{2}}$, respectively. Let $\pi_{1}:=\{n ; n \neq \equiv 1 \bmod 4\}$ and $\pi_{2}:=\{n ; n \neq \equiv 3 \bmod 4\}$. Both of these sets are II-codings and correspond to the following game diagrams:

$$
\begin{aligned}
& \begin{array}{rrrrrr}
\mathcal{G}^{\pi_{1}, \mathrm{II}} & x_{0} & & x_{2}, x_{3}, x_{4} & x_{6}, x_{7}, x_{8} & \cdots \\
\text { II } & & & x_{1} & x_{5} & x_{9}
\end{array} \\
& \mathcal{G}^{\pi_{2}, \text { II }} \mathrm{I} x_{0}, x_{1}, x_{2} \quad x_{4}, x_{5}, x_{6} \quad x_{8}, x_{9}, x_{10} \quad \ldots \\
& \text { II } x_{3} \quad x_{7} \quad x_{11} \ldots
\end{aligned}
$$

By Lemma 11, there are optimal strategies $\left(\tau_{1}\right)_{\pi_{1}},\left(\tau_{2}\right)_{\pi_{2}}$ for player II in $\mathcal{G}_{A_{1}}^{\pi_{1} \text { II }}$ and $\mathcal{G}_{A_{2}}^{\pi_{2} \text {,II }}$, respectively. To reduce notation, we write $\tau_{1}^{*}:=\left(\tau_{1}\right)_{\pi_{1}}$ and $\tau_{2}^{*}:=\left(\tau_{2}\right)_{\pi_{2}}$. We combine these strategies into an optimal strategy $\tau$ in $\mathcal{G}_{A_{1} \cap A_{2}}$ by

$$
\begin{aligned}
\tau\left(x_{0}, \ldots, x_{4 n}\right) & :=\tau_{1}^{*}\left(x_{0}, \ldots, x_{4 n}\right), \\
\tau\left(x_{0}, \ldots, x_{4 n+2}\right) & :=\tau_{2}^{*}\left(x_{0}, \ldots, x_{4 n+2}\right) .
\end{aligned}
$$

We show that $\tau$ is optimal, by letting $\sigma$ be arbitrary for player $\operatorname{I}$ in $\mathcal{G}_{A_{1} \cap A_{2}}$, and define strategies $\sigma_{1}$ and $\sigma_{2}$ in $\mathcal{G}_{A_{1}}^{\pi_{1}, \text { II }}$ and $\mathcal{G}_{A_{2}}^{\pi_{2} \text {,II }}$, respectively:

$$
\begin{aligned}
\sigma_{1}(\varnothing) & :=\sigma(\varnothing), \\
\sigma_{1}\left(x_{0}, \ldots, x_{2 n+1}\right) & :=\sigma\left(x_{0}, \ldots, x_{2 n+1}\right), \\
\sigma_{1}\left(x_{0}, \ldots, x_{4 n+2}\right) & :=\tau_{2}^{*}\left(x_{0}, \ldots, x_{4 n+2}\right), \\
\sigma_{2}\left(x_{0}, \ldots, x_{2 n+1}\right) & :=\sigma\left(x_{0}, \ldots, x_{2 n+1}\right), \\
\sigma_{2}\left(x_{0}, \ldots, x_{4 n}\right) & :=\tau_{1}^{*}\left(x_{0}, \ldots, x_{4 n}\right),
\end{aligned}
$$

Then it is easy to check that $\mu_{\sigma, \tau}=\mu_{\sigma_{1}, \tau_{1}^{*}}=\mu_{\sigma_{2}, \tau_{2}^{*}}$ and thus

$$
\mu_{\sigma, \tau}\left(\left\{\vec{x} ; \operatorname{ran}(\vec{x}) \in A_{1}\right\}\right)=\mu_{\sigma_{1}, \tau_{1}^{*}}\left(\left\{\vec{x} ; \operatorname{ran}(\vec{x}) \in A_{1}\right\}\right)=1,
$$

and similarly for $A_{2}$, and thus $\tau$ is optimal.

$\square($ Claim 13) 
Claim 14 For a family $\left\{A_{x} ; x \in \mathbb{R}\right\} \subseteq U$, the diagonal intersection $\triangle_{x \in \mathbb{R}} A_{x}$ is in $U$.

Proof (of Claim 14) As in [18], this is the most intricate part of the proof.

Subclaim 15 There is a choice function $x \mapsto \tau_{x}$ picking an optimal strategy for $\mathcal{G}_{A_{x}}$.

Proof (of Subclaim 15) The argument is exactly the same as the one in the proof of normality of $U$ in the last section.

Fix a bookkeeping bijection $\rho$ from $\omega \times \omega$ to $\omega$ such that $\rho(n, m)<\rho(n, m+1)$ and $\rho(n, 0) \geq n$. We are playing infinitely many games in a diagram where the first coordinate is for the index of the game we are playing, and the second coordinate is for the number of moves. Hence the pair $(n, m)$ stands for " $m$ th move in the $n$th game". Define a II-coding $\pi_{n}:=\omega \backslash\{2 \rho(n, i)+1 ; i \in \omega\}$ corresponding to the following game diagram:

$$
\begin{aligned}
& \text { I } x_{0}, \ldots, x_{2 \rho(n, 0)} x_{2 \rho(n, 0)+2}, \ldots, x_{2 \rho(n, 1)} \\
& \text { II }
\end{aligned} x_{2 \rho(n, 0)+1} \quad \cdots
$$

By Lemma 11 and Subclaim 15, we know that for each $x$, we get an optimal strategy $\left(\tau_{x}\right)_{\pi_{n}}$ for the game $\mathcal{G}_{A_{x}}^{\pi_{n}, \text { II }}$. Let $\tau$ be the following mixed strategy

$$
\tau\left(x_{0}, \ldots, x_{2 \rho(n, m)}\right):=\left(\tau_{x_{n}}\right)_{\pi_{n}}\left(x_{0}, \ldots, x_{2 \rho(n, m)}\right) .
$$

The properties of $\rho$ make sure that this strategy is well-defined; we shall now prove that $\tau$ is an optimal strategy for player II in $\mathcal{G}_{\triangle_{x \in \mathbb{R}}} A_{x}$.

Pick any mixed strategy $\sigma$ for player I in $\mathcal{G}_{\triangle_{x \in \mathbb{R}}} A_{x}$, and define strategies $\sigma_{n}$ for $\mathcal{G}^{\pi_{n}, \text { II }}$. Let $m=\rho(k, \ell)$, then

$$
\begin{aligned}
\sigma_{n}\left(x_{0}, \ldots, x_{2 m-1}\right) & :=\sigma\left(x_{0}, \ldots, x_{2 m-1}\right), \text { and } \\
\sigma_{n}\left(x_{0}, \ldots, x_{2 m}\right) & :=\left(\tau_{x_{k}}\right)_{\pi_{k}}\left(x_{0}, \ldots, x_{2 m}\right)(\text { if } k \neq n) .
\end{aligned}
$$

Note that for each $n \in \omega$ and $s \in{ }^{n+1} \mathbb{R}, \mu_{\sigma, \tau}$ and $\mu_{\sigma_{n},\left(\tau_{s(n)}\right)_{\pi_{n}}}$ agree below [s], i.e., for any set $A \subseteq[s], \mu_{\sigma, \tau}(A)=\mu_{\sigma_{n},\left(\tau_{s(n)}\right)_{\pi_{n}}}(A)$.

The payoff set (for player II) for $\mathcal{G}_{\triangle_{x \in \mathbb{R}}} A_{x}$ is $A:=\left\{\vec{x} ; \operatorname{ran}(\vec{x}) \in \triangle A_{x}\right\}$. We show that $\mu_{\sigma, \tau}(A)=1$. Since

$$
A=\bigcap_{n \in \omega}\left\{\vec{x} ; \operatorname{ran} \vec{x} \in A_{x_{n}}\right\}
$$

it suffices to check that the sets $A_{n}:=\left\{\vec{x} ; \operatorname{ran}(\vec{x}) \in A_{x_{n}}\right\}$ has $\mu_{\sigma, \tau}$-measure 1 . for all $n \in \omega$. But $\mu_{\sigma_{n},\left(\tau_{s(n)}\right)_{\pi_{n}}}\left([s] \cap A_{n}\right)=\mu_{\sigma_{n},\left(\tau_{s(n)}\right)_{\pi_{n}}}([s])$. Then, using the fact that the measures have countable support, we get

$$
\begin{aligned}
\mu_{\sigma, \tau}\left(A_{n}\right) & =\sum_{s \in \in^{n+1} \mathbb{R}} \mu_{\sigma, \tau}\left([s] \cap A_{n}\right) \\
& =\sum_{s \in \in^{n+1} \mathbb{R}} \mu_{\sigma_{n},\left(\tau_{s(n)}\right)_{\pi_{n}}}\left([s] \cap A_{n}\right)
\end{aligned}
$$




$$
\begin{aligned}
& =\sum_{s \in \in^{n+1}[\mathbb{R}} \mu_{\sigma_{n},\left(\tau_{s(n)}\right)_{\pi_{n}}}([s]) \\
& =\sum_{s \in \in^{n+1} \mathbb{R}} \mu_{\sigma, \tau}([s]) \\
& =1 .
\end{aligned}
$$

$\square($ Claim 14)

Note that together with the trivial properties of $U$ mentioned at the beginning of this section, Claims 12, 13, and 14 are all we need to show: The non-principality of $U$ follows from the fineness of $U$, and the $\sigma$-completeness follows from the fact that every set of reals is Lebesgue measurable (an ultrafilter failing $\sigma$-completeness defines a non-principal ultrafilter on $\omega$ and hence a non-Lebesgue measurable set). Thus, we have finished the second proof of the Main Theorem.

\section{Conclusion: further results and open questions}

\subsection{The consistency strength of $B I-A D_{\mathbb{R}}$}

Main Theorem 1 shows that the consistency strength of $B I-A D_{\mathbb{R}}$ is strictly greater than that of BI-AD. In unpublished work, Woodin and the first author made steps towards computing the exact consistency strength of $B I-A D_{\mathbb{R}}[5]$. They conjecture that $B I-A D_{\mathbb{R}}$ and $A D_{\mathbb{R}}$ are equiconsistent [5, Conjecture 6.2] and show that this conjecture follows from plausible inner model theoretic statements.

The following argument gives an indication of what lower bounds we currently know how to prove: We give a sketch of a proof of $A D^{L}\left(\mathbb{R}, \mathbb{R}^{\#}\right)$ from $B I-A D_{\mathbb{R}}$. This is a stronger assumption than $A D^{\mathbf{L}(\mathbb{R})}+$ " $\mathbb{R}^{\#}$ exists". 7

By Theorems 8 and 1 , we have $A D^{\mathbf{L}(\mathbb{R})}$ and that $\mathbb{R}^{\#}$ exists. By a result of Solovay (cf. [17, p. 117]), this implies that every set of reals in $\mathbf{L}(\mathbb{R})$ is $\operatorname{Suslin}$ in $\mathbf{L}\left(\mathbb{R}, \mathbb{R}^{\#}\right)$. If we let $W_{n, m}$ be the set of $\Sigma_{n}$ formulas true in $\mathbf{L}(\mathbb{R})$ with $m$ indiscernibles, and $F_{n, m}$ the set of $\Sigma_{n}$ formulas false in $\mathbf{L}(\mathbb{R})$ with $m$ indiscernibles, then each of these sets is in $\mathbf{L}(\mathbb{R})$ and thus Suslin, and furthermore, $\mathbb{R}^{\#}=\bigcup_{n, m \in \omega} W_{n, m}$ and $\mathbb{R} \backslash \mathbb{R}^{\#}=\bigcup_{n, m \in \omega} F_{n, m}$. Assuming $A C_{\mathbb{R}}(\mathbb{R})$ in $\mathbf{V}$, we have $\mathrm{DC}$ in $\mathbf{L}\left(\mathbb{R}, \mathbb{R}^{\#}\right)$ because every set in $\mathbf{L}\left(\mathbb{R}, \mathbb{R}^{\#}\right)$ can be coded by an ordinal, a real and $\mathbb{R}^{\#}$. We use DC to pick witnesses for the Suslinness of $W_{n, m}$ and $F_{n, m}$ and can thus show that $\mathbb{R}^{\#}$ is both Suslin and co-Suslin.

Let $(\$)$ denote the statement "all sets that are both Suslin and co-Suslin are determined"; in [7, Theorem 1.2], Kechris and Woodin show that ( $\$$ ) implies that $\mathbf{L}(\mathbb{R}) \models A D$. For any set $X$ that is both Suslin and co-Suslin, this easily generalizes to $\mathbf{L}(\mathbb{R}, X) \models A D$. But we assumed $B \mathrm{AD}_{\mathbb{R}}$, and thus by [15, Lemma 4.1], we get that ( $\ddagger)$ holds. Collecting all of the components, we obtain $\mathbf{L}\left(\mathbb{R}, \mathbb{R}^{\#}\right) \models A D$.

\footnotetext{
7 By results of Steel, $\mathbf{L}\left(\mathbb{R}, \mathbb{R}^{\#}\right) \models A D$ is equiconsistent to ZFC + "there is a limit of Woodin cardinals $\lambda$ such that for all $x \in \mathbf{V}_{\lambda}$, the set $\mathbf{M}_{\omega}^{\#}(x)$ exists". The authors would like to thank Hugh Woodin who pointed out the proof idea to the first author at a meeting in Vienna in 2009.
} 
The same argument can now be applied to $\mathbf{L}\left(\mathbb{R}, \mathbb{R}^{\#}\right)$ to give $A D^{\mathbf{L}\left(\mathbb{R}, \mathbb{R}^{\# \#}\right)}$, or more inductively.

\subsection{Getting a fine measure from BI-AD}

It is well-known that Solovay's proof of the existence of a fine normal measure on $\wp_{\omega_{1}}(\mathbb{R})$ under $A D_{\mathbb{R}}$ can be modified to work under $A D$ by a simple coding argument (cf. $[8, \S 4.5]$ ) if you give up normality, thus giving a proof of the following theorem:

Theorem 16 If $\mathrm{AD}$ holds, then $\omega_{1}$ is $\kappa$-strongly compact for every $\kappa<\Theta$.

Our proofs in Sects. 4 and 5 do not seem to allow us to prove the Blackwell analogue of Theorem 16 (essentially, since the modified games are not range invariant anymore, as they rely on the coding used).

\section{$6.3 \mathrm{BI}-\mathrm{AD}_{\mathbb{R}}$ and the cofinality of $\Theta$}

Solovay proved that the consistency strength of $A D_{\mathbb{R}}+\operatorname{cf}(\Theta)>\omega$ is strictly bigger than that of $A D_{\mathbb{R}}$. This proof uses model constructions based on the Wadge hierarchy (cf. [18, Theorems 2.5 and 5.7]). In order to prove the appropriate analogues in the Blackwell context, we should need to make use of the Blackwell analogue of the Wadge hierarchy, the Blackwell Wadge hierarchy (cf. [12, § 7.3]). Unfortunately, we do not know how to prove that this hierarchy is wellfounded (not even under the assumption of $\left.\mathrm{Bl}-\mathrm{AD}_{\mathbb{R}}\right)$.

Acknowledgments The research of the first author was supported by a GLoRiClass fellowship funded by the European Commission (Early Stage Research Training Mono-Host Fellowship MEST-CT-2005020841) and a grant by the Japan Society for the Promotion of Science; the work of the second author was done while he was at the Vrije Universiteit Amsterdam; the work of the third author was partially supported by the DFG-NWO Bilateral Grant KO 1353-5/1/DN 62-630. The authors should like to thank Vincent Kieftenbeld (Edwardsville IL) for discussions in the early phase of this project. They are grateful to an anonymous referee for suggesting a simpler proof of Lemma 11.

Open Access This article is distributed under the terms of the Creative Commons Attribution License which permits any use, distribution, and reproduction in any medium, provided the original author(s) and the source are credited.

\section{References}

1. Becker, H.: AD and the supercompactness of $\aleph_{1}$. J. Symb. Logic 46(4), 822-842 (1981)

2. Blackwell, D.: Infinite $G_{\delta}$ games with imperfect information. Polska Akademia Nauk-Instytut Matematyczny-Zastosowania Matematyki 10, 99-101 (1969)

3. Blackwell, D.: Games with infinitely many moves and slightly imperfect information. In: Nowakowski, R.J. (eds.) Games of No Chance, Combinatorial Games at MSRI, Workshop, July 11-21, 1994 in Berkeley, CA, USA, Mathematical Sciences Research Institute Publications, vol. 29, pp. 407-408 (1997)

4. Gale, D., Stewart, F.M.: Infinite games with perfect information. In: Kuhn, H.W., Tucker, A.W. (eds.) Contributions to the Theory of Games II, Annals of Mathematical Studies, vol. 28, pp. 245-266 (1953)

5. Ikegami, D., Woodin, W.H.: Real determinacy and real Blackwell determinacy. Institut Mittag-Leffler Report 32, 2009/10 (2010) 
6. Kanamori, A.: The Higher Infinite, Large Cardinals in Set Theory from their Beginnings Perspectives, in Mathematical Logic. Springer, Berlin (1994)

7. Kechris, A.S., Woodin, W.H.: The equivalence of partition properties and determinacy. In: Kechris, A.S., Löwe, B., Steel, J.R. (eds.) Games, Scales, and Suslin Cardinals: The Cabal Seminar, vol. I, Lecture Notes in Logic, vol. 31, pp. 355-378 (2008)

8. Kieftenbeld, V.: Notions of strong compactness without the axiom of choice. Doctoraalscriptie Wiskunde, Universiteit van Amsterdam, ILLC Publications PP-2006-22 (2006)

9. de Kloet, D.: Real Blackwell Determinacy. ILLC Publications X-2005-05 (2005)

10. Löwe, B.: Playing with mixed strategies on infinite sets. Int. J. Game Theory 31, 137-150 (2002)

11. Löwe, B.: The simulation technique and its consequences for infinitary combinatorics under the axiom of Blackwell determinacy. Pac. J. Math. 214, 335-358 (2004)

12. Löwe, B.: Set theory of infinite imperfect information games. In: Andretta, A. (ed.) Set Theory: Recent Trends and Applications, Quaderni di Matematica, vol. 17, pp. 137-181. Napoli (2005)

13. Martin, D.A.: The determinacy of Blackwell games. J. Symb. Logic 63, 1565-1581 (1998)

14. Martin, D.A.: A simple proof that determinacy implies Lebesgue measurability. Rendiconti del Seminario Matematico Dell' Università e di Polytechnico di Torino 61, 393-397 (2003)

15. Martin, D.A., Neeman, I., Vervoort, M.: The strength of Blackwell determinacy. J. Symb. Logic 68, 615-636 (2003)

16. Martin, D.A., Steel, J.R.: The extent of scales in $\mathbf{L}(\mathbb{R})$. In: Kechris, A.S., Martin, D.A., Moschovakis, Y.N. (eds.) Cabal Seminar 79-81. Proceedings of the Caltech-UCLA logic seminar held during the academic years 1979/1981, Lecture Notes in Mathematics, vol. 1019, pp. 86-96. Springer, Berlin (1983)

17. Martin, D.A., Steel, J.R.: The extent of scales in $\mathbf{L}(\mathbb{R})$. In: Kechris, A.S., Löwe, B., Steel, J.R. (eds.) Games, Scales, and Suslin Cardinals: The Cabal Seminar, vol. I, Lecture Notes in Logic, vol. 31, pp. 110-120. Cambridge University Press, Cambridge (2008). Reprint of [16]

18. Solovay, R.M.: The independence of DC from AD. In: Kechris, A.S., Moschovakis, Y.N. (eds.) Cabal Seminar 76-77. Proceedings of the Caltech-UCLA Logic Seminar 1976-77, Lecture Notes in Mathematics, vol. 689, pp. 171-183. Springer, Berlin (1978)

19. Vervoort, M.: Blackwell games. In: Ferguson, T.S., Shapley, L.S., MacQueen, J.B.(eds.) Statistics, Probability, and Game Theory: Papers in Honor of David Blackwell, Institute of Mathematical Statistics Lecture Notes-Monograph Series, vol. 30, pp. 369-390 (1996) 